\title{
Pinwheel Patterns: From 2D to 3D Schemas
}

\author{
Christopher F. Earl $^{1} \cdot$ Iestyn Jowers $^{1}$
}

Published online: 18 August 2015

(C) Kim Williams Books, Turin 2015

\begin{abstract}
Pinwheels are generic configurations in architectural layout planning. Planar pinwheels provide familiar schemes for layouts which present design 'in the round' with a cyclic symmetry. The paper examines the 3-D versions of 2-D pinwheels where a 'locked' joint with three rectangular volume elements aligned along orthogonal axes is a characteristic feature. Pairing handed versions of these locked joints yields a candidate for a 3-D pinwheel schema with six repeated volume elements and threefold cyclic symmetry. Shape rules, based on spatial relations between volumes, generate this and other examples of 3-D pinwheel schemas. These schemas are set in a wider analysis of the numbers and types of joints in 3 -rectangulations in terms of maximal bounding planes. The bounding-plane views of the arrangements is set alongside more functional volume descriptions which enables the elements and relations in architectural form to be (re)generated and (re)interpreted both 'in view' and 'in use'.
\end{abstract}

Keywords Pinwheel $\cdot$ Rectangulation - Spatial relation - Shape rule · Schema

\section{Introduction}

Pinwheels are commonly manifested as spiral or handed arrangements of architectural elements. In their purest form they exhibit a cyclic symmetry where a shape element is repeated by rotating it incrementally to produce a characteristic

Christopher F. Earl

c.f.earl@open.ac.uk

Iestyn Jowers

i.r.jowers@open.ac.uk

1 Department of Engineering and Innovation, Open University, Milton Keynes MK7 6AA, UK 
Fig. 1 Pinwheel pattern (Serlio 1545: 21v)
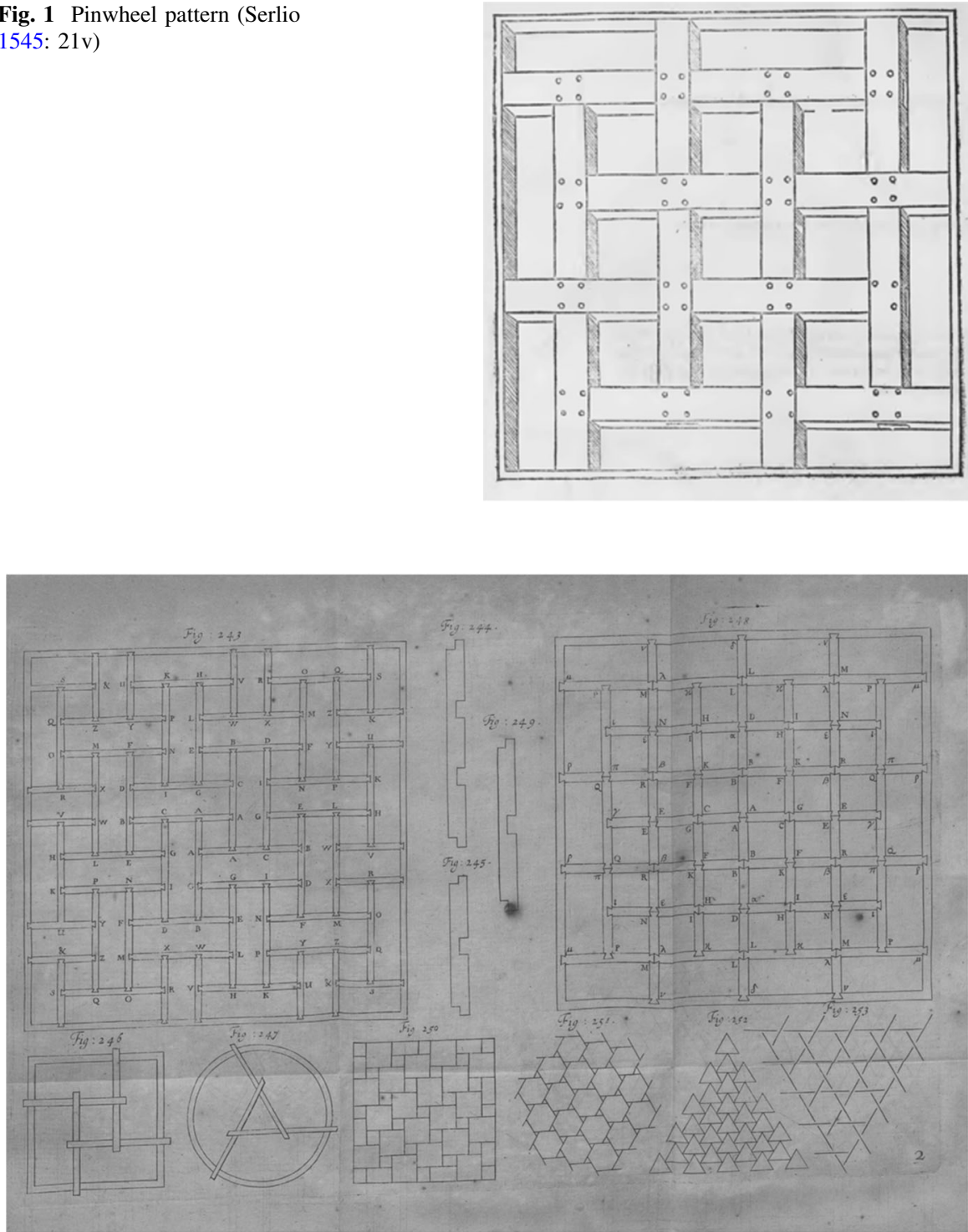

Fig. 2 Pinwheel patterns from Mechanica (Wallis 1670: unnumbered plate)

'spiral' arrangement. Patterns of this type are widespread and diverse in architecture where the underlying pinwheel schema of a repeated spatial relation appears in twodimensional plans and structures as well as three-dimensional compositions of floors, walls and spatial volumes. This paper examines these three-dimensional generalisations with calculations of dependencies among spatial relations of different types and shape rules used to generate them. 
A prevalent example of a pinwheel occurs when four elements spin around a central void. It is used structurally, decoratively and architecturally. Serlio (1545: $21 \mathrm{v})$ appears to have been instrumental in proposing pinwheel arrangements in floor/ceiling structures for large spans (Fig. 1). Serlio's potential influence is analysed by Yeomans (1997: 74-83) including examples in near contemporary English Tudor mansions such as Wollaton Hall (Yeomans 1997: 76) and in the geometrical and mechanical investigations (Fig. 2) by John Wallis who was Savilian Professor of Geometry at Oxford between 1649 and 1703 (Wallis 1670: unnumbered plate). He was a possible influence on Christopher Wren, who as Savilian Professor of Astronomy between 1661 and 1673, developed designs for the roof of the Sheldonian Theatre in Oxford. Initial designs appear to incorporate patterns developed by Serlio and Wallis for long spans without supporting columns, although the realized design used composite trusses rather than pinwheel patterns.

The paper traces the development of these pinwheel arrangements in architecture and design. In the first part it analyses the ways that the arrangements were used in both two- and three-dimensional configurations of architectural elements; floors, walls and the spaces they bound. The second part considers three-dimensional examples and their constituent spatial relations in more detail and the third part sets up some notation for describing and calculating the distribution of different spatial relations in three-dimensional spatial arrangements. Finally the paper develops compact examples of three-dimensional pinwheel patterns analogous to the twodimensional patterns of Wallis and Serlio. Repeating shape rules generate these patterns that form schemas for three-dimensional architectural arrangements of interlocking elements.

\section{Pinwheels in Architecture and Design}

Architectural compositions of plane segments and volume elements on an orthogonal grid are widespread and this paper focuses on corresponding 2- and 3-D pinwheels. Common pinwheel type arrangements in 2- and 3-dimensions derive from spatial relations between 3-rectangles or cuboids, respectively, as shown in Fig. 3. The 2-D arrangement repeats a spatial relation four times with resulting $\mathrm{C}_{4}$ cyclic symmetry. The 3-D-pinwheel repeats a spatial relation three times and yields a threefold $\mathrm{C}_{3}$ symmetry about a diagonal axis on the orthogonal grid. The result is a closely interlocked, or 'locked' joint, between elements.

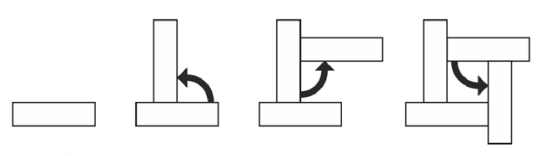

(a)

Fig. 3 Spatial relations in a 2-D pinwheels and $\mathbf{b}$ 3-D pinwheels

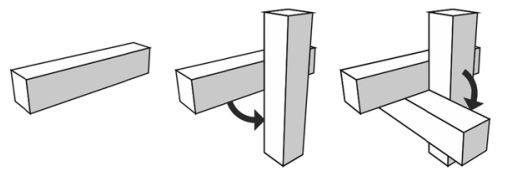

(b) 


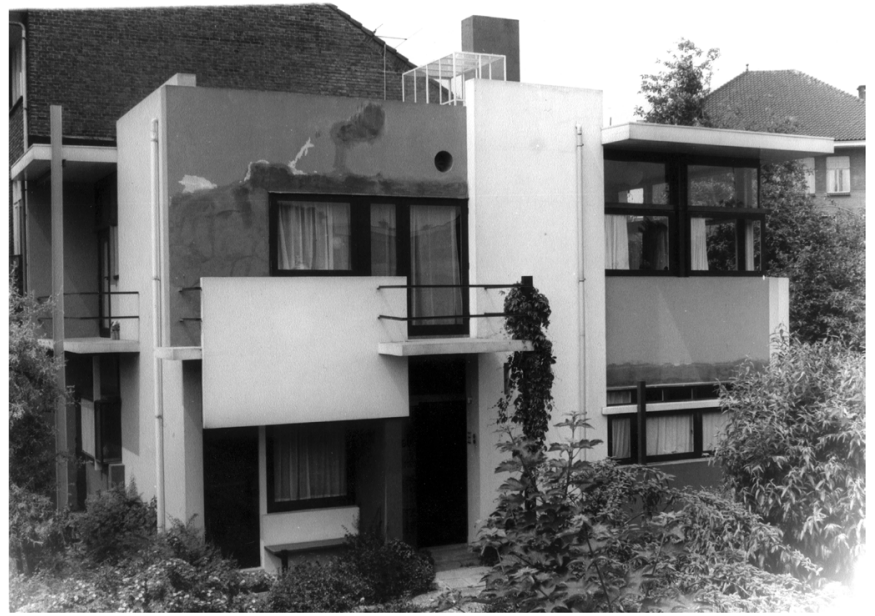

(a)

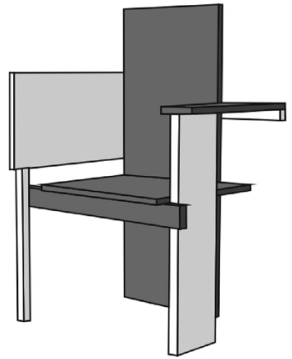

(b)

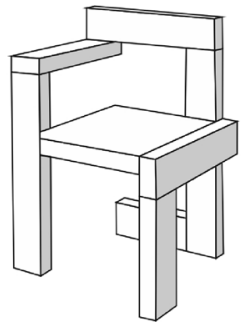

(c)

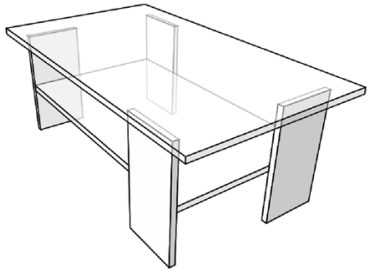

(d)

Fig. 4 a Schroeder House, b Berlin chair, c Steltman chair and d a table with a 2-D pinwheel arrangement for its supports. Images generated by the authors

Designers and architects have used these pinwheel arrangements in several contexts. In the twentieth-century artists such as Van Doesberg (1969) developed abstract spatial configurations whilst designers such as Rietveld (Dettingmeijer et al. 2010), applied them extensively in his furniture and houses. Colour, textures and functional architectural attributes add further dimensions to these compositions by highlighting selected elements and relations. The explorations, by Rietveld (Fig. $4 \mathrm{a}-\mathrm{c}$ ) of spatial relations among 3-D rectangles used a wide variety of joints between elements including the 'locked' joints. These latter serve to emphasise the three-dimensional character of the designs.

The Schroeder house displays some elements of a pinwheel plan with its central 'spiral' staircase as well as the 3-D composition of rectangular slabs articulating the interior (and exterior) spaces. The same effect is created in the chairs (Fig. 4b, c) with structural rectangular elements, composed in face-to-face relations. These designs can be viewed variously as arrangements of (1) 3-D 'planes' (or thin cuboids), for walls and roofs bound the volumes of spaces and rooms or (2) of these 

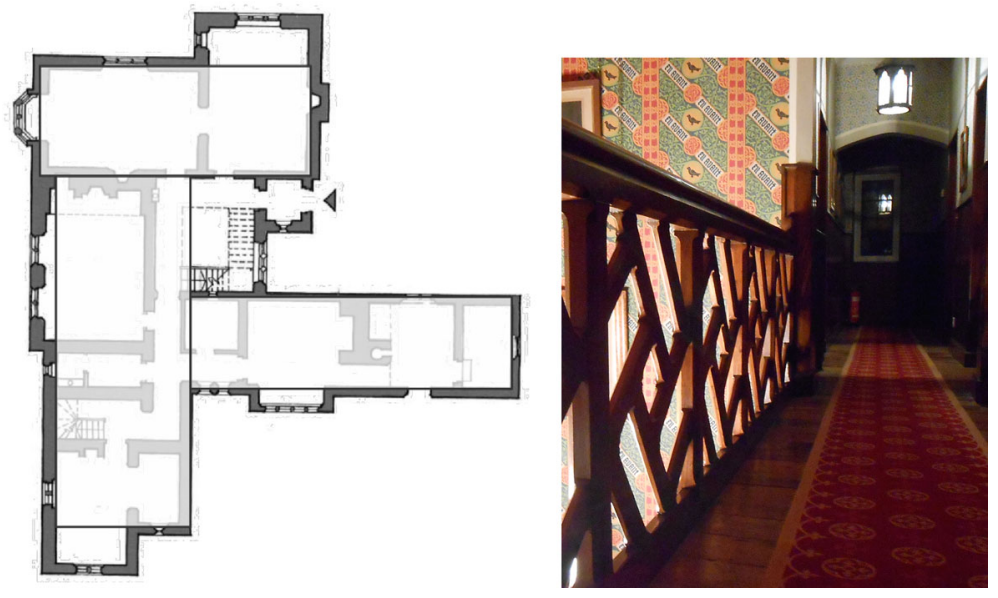

Fig. 5 The Grange, Ramsgate, (Pugin A W N) overall pinwheel plan and pinwheel decorative baluster. Images generated by authors

volumes directly. For the Schroeder House it is relevant to observe that the fluid structure of internal architectural spaces, with several retractable or folding screens, is set alongside the well defined 3-D 'planes' used to articulate roofs, walls and floors

In the next section general spatial arrangements of 3-rectangle volumes and their bounding planes are analysed in terms of spatial relations, including 3-D locked joints and pinwheels. Before doing this historical examples are described of how pinwheels provide tight spatial unity in architectural layouts especially in presenting design 'in the round' such as point blocks and individual dwellings. In the twentieth-century both Wright (March and Steadman 1971) and Schindler were exponents of the cyclic plan with clear nineteenth-century precursors in the architecture of the Gothic revival and the arts and crafts movements. For example Pugin's domestic designs draw heavily on these cyclic configurations. BrittainCatlin (2004: 101-105) discusses how Pugin's pinwheel plans eschew bilateral symmetry in favour of a cyclic symmetry. Figure 5 shows an example in the Grange at Ramsgate, Pugin's own home. Three of the four elements in the pinwheel are picked out in the overall layout shown in Fig. 5 and the central staircase winds upwards with the spiral of the pinweel in the baluster woodwork (Fig. 5). An analysis (Brittain-Catlin 2004: 107) of Pugin's (now demolished) Bishop's Palace in Birmingham displays a 3-D pinwheel arrangement that provides an intricate processional route climbing from the entrance to the main reception/audience rooms through a sequence of stairs and corridors.

\section{Orthogonal 3D Configurations and their Spatial Relations}

Among spatial relations between volume elements, face adjacencies represent sites for functional connections in architecture, structural design and spatial layout of engineering designs. This paper deals with 3-rectangulations or orthogonal 3D 
configurations of cuboids (3-rectangles). A key distinction is made (Krishnamurti and Earl 1998) between 3-rectangulations containing 'locked' 3-D pinwheels (Fig. 3) and those without (termed 'unlocked' rectangulations). A further distinction is made between densely packed 3-rectangulations that have no internal voids and loosely packed ones. 3-rectangulations are analysed (Krishnamurti and Earl 1998) in terms of the maximal planes composed from the boundaries of cuboids. In Fig. 6 a loosely packed 3-rectangulation with six $1 \times 1 \times 3$ rectangles has a central cubical void. There are locked pinwheel joints at each of the eight joints between 3 -rectangles around the central void with 'internal' maximal planes shaped as an $\mathbf{H}$ and 'external' ones shaped in a + .

Face-adjacency is constrained in densely packed, unlocked 3-rectangulations (Krishnamurti and Earl 1998) with a rectangular boundary. In this case maximal planes are themselves rectangular. A representation of adjacencies between maximal planes as a directed map has no 3-cycles and no other cycles that include planes in all three directions. This lack of cycles containing maximal planes in all three directions is a characteristic property of unlocked 3-rectangulations that are essentially two-dimensional in character. It is the locked pinwheel patterns that exemplify fully three-dimensional arrangements.

In order to explore general configurations of face-adjacent 3-rectangles in loosely packed arrangements, the joints between cuboids are classified according to their incident maximal planes (Earl 1978). Joints can be classified as three types. Type 1 has three maximal planes, one having a convex corner at the joint. Type 2 has three planes each having a convex corner, and type 3 is the 'locked' joint with three maximal planes and three concave corners. This paper considers these configurations of face-adjacent 3-rectangles with joints of types 1, 2 and 3. Those with only joints of type 1 and 2 are called unlocked and those with type 3 joints are locked. Figure 7 shows examples of these three types. The types of joint are distinguished by their subscripts. Type 1 joints $X_{1}$ and $C_{1}$ have one convex and one concave corner respectively on the maximal planes. Type 2 joints $X_{2}$ and $C_{2}$ have two convex and two concave corners respectively, whilst the pinwheel joint $C_{3}$ has three concave corners and the external corner $X_{3}$ has three convex corners. Figure 7 shows examples of these joints that are adjacent to exterior or internal voids,
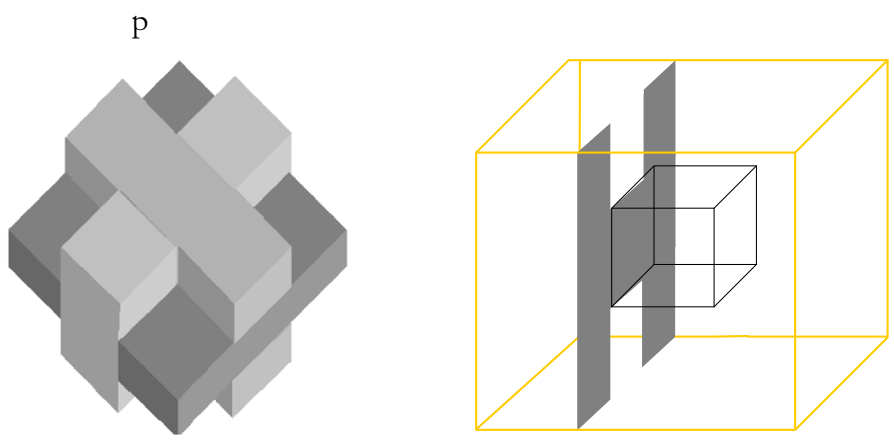

Fig. 6 A 3-rectangulation with six equal 3-rectangles 

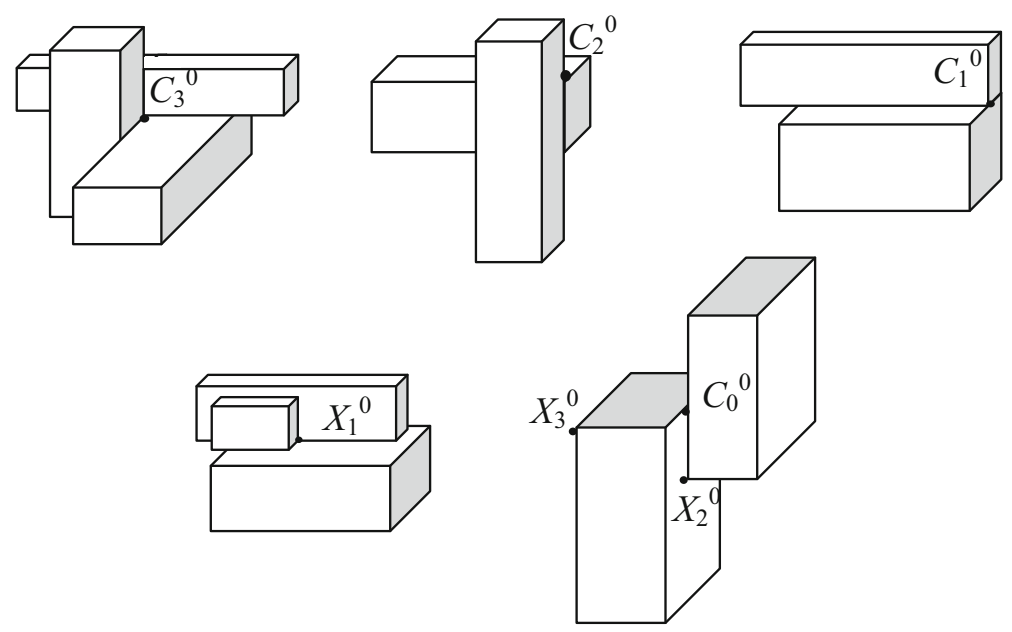

Fig. 7 Type 1 joints $\left(X_{1}^{0}\right.$ and $C_{1}^{0}$ plus $\left.C_{0}^{0}\right)$, type $2\left(X_{2}^{0}\right.$ and $\left.C_{2}^{0}\right)$ and type $3\left(X_{3}^{0}\right.$ and $\left.C_{3}^{0}\right)$

indicated by the 'zero' superscripts $X^{0}$ and $C^{0}$. The joint $C_{0}^{0}$ is distinguished as a special case of a type 1 joint at the partial face adjacency of just two 3-rectangles.

In the joints shown in Fig. $7, C_{3}^{0}$ has three concave corners of maximal planes and is also counted on three convex corners of 'exterior' planes. These exterior planes may not be maximal planes. $C_{2}^{0}$ has concave corners of two maximal planes and is also counted on one concave corner and two convex corners of exterior planes. $C_{1}^{0}$ has a concave corner of one maximal plane and a convex corner of one maximal plane and is also counted on one concave corner and two convex corners of exterior planes. $X_{1}^{0}$ has a convex corner of one maximal plane and is on convex corners of two exterior planes and a concave corner of a third. $X_{2}^{0}$ is not on the concave corner of maximal plane but on convex corners of two exterior (and maximal) planes and the concave corner of one exterior plane. $X_{3}^{0}$ has convex corners of three maximal planes all exterior. $C_{0}^{0}$ has one concave corner on a maximal plane and two convex corners of exterior planes.

Types $C_{3}^{0}, C_{2}^{0}, C_{1}^{0}$ have concave corners of three, two and one maximal planes respectively at the joint. Types $X_{3}^{0}, X_{2}^{0}, X_{1}^{0}$ have three, two and one convex corners of maximal planes, respectively, at the joint. Each exterior joint is at the corner of exactly one 3-rectangle except for type $C_{0}^{0}$ and type $C_{3}^{0}$ when the joint is open to the outside on both sides (in which case it is not at the corner of any cuboid) or interior locked joint (when it is at the corners of two cuboids). The locked configuration of just three rectangles in Fig. 4 has a $C_{3}^{0}$ joint with no cuboid corners. Note that interior joints are only of types $X_{1}$ or $C_{3}$ that are both at the corners of two 3-rectangles. Arrangements of four 3-rectangles clustered at a joint, where two maximal planes cross, are excluded. Maximal planes do not have corners, either convex or concave, at such joints. 

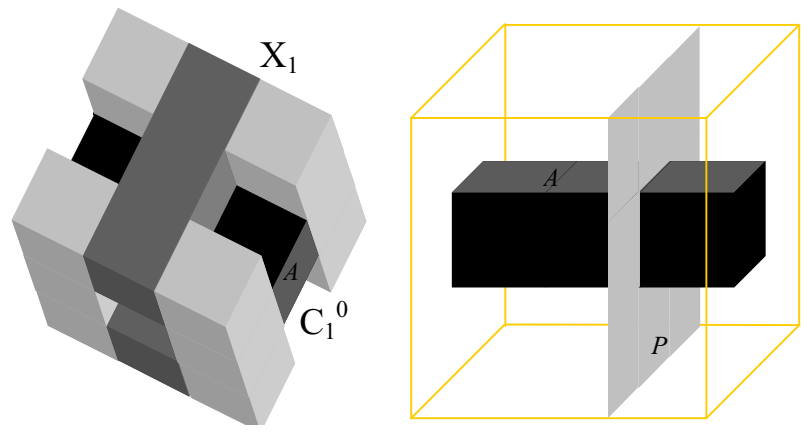

Fig. 8 Locked 3-rectangulation with a 'through' cuboid A. Maximal planes, both interior and boundary, have either $\square$, $\mathrm{H}$ or + shapes
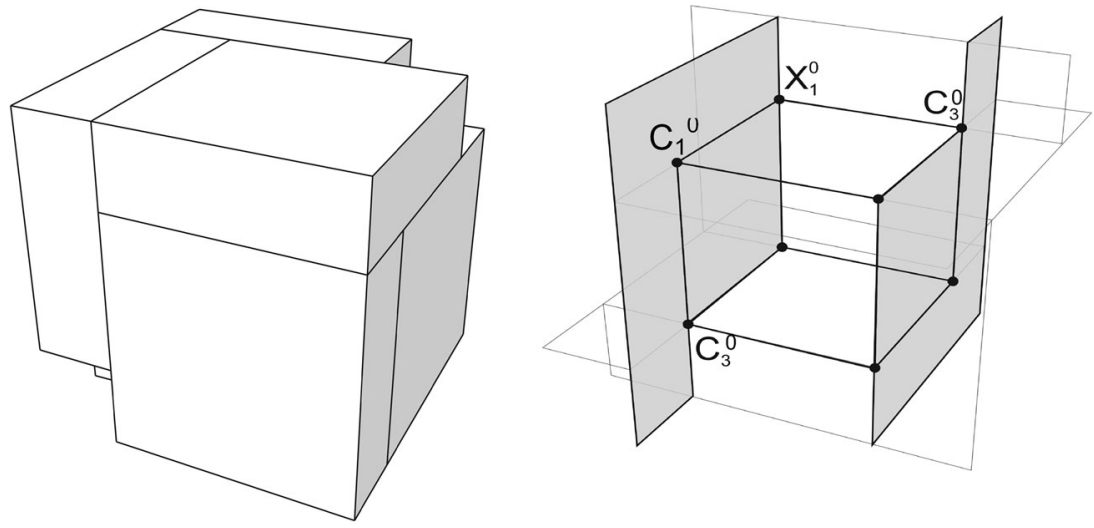

Fig. 9 A 3-rectangulation with six equal cuboids, a central void, two concave corners and internal L-shaped maximal planes

To conclude this examination of cuboids, joints and maximal planes in 3rectangulations two further examples are presented, before developing an analysis of dependencies among the numbers of different types of joints in the next section. Maximal planes $P$ in the locked 3-rectangulation of Fig. 8 have holes created by a 'through' cuboid $A$. All the eight 'interior' joints are locked $C_{3}^{0}$ pinwheels and the 'exterior' joints are types $C_{1}^{0}$ or $X_{1}$.

The second example is drawn with an overall shape bounded by a $4 \times 4 \times 4$ cube (Fig. 9). It consists of six $3 \times 3 \times 1$ cuboids aligned face to edge. Obvious symmetries of the shape are the rotations about the diagonal axes of the cube and a central inversion. The six cuboids surround a central $2 \times 2 \times 2$ internal void. Each maximal plane is L-shaped. Effectively this configuration is formed from a pair of 3-D locked pinwheels $C_{3}^{0}$.

The cyclic shape in Fig. 9 is similar to a composition in Froebel's sixth gift (that uses $2 \times 2 \times 1$ cuboids) and may have contributed to Wright's exercises in spatial 
composition (MacCormac 1974; Stiny 1980) The geometric and generative analysis of general compositions of face adjacent cuboids complements previous analysis (Krishnamurti and Earl 1998) of 3-rectangulations with 'unlocked' joints that have an underlying two-dimensional structure. The locked rectangulations are essentially three-dimensional.

The compact examples in Figs. 6, 8 and 9 present 3-D architectural schemas in three dimensions that are analogous to the 2-D pinwheels of Serlio and Wallis. Each of these schemas is generated by repetitions of spatial relations using shape rules (Stiny 1980). The next section provides generative descriptions.

\section{Generative Descriptions of Pinwheel Schemas}

The 3-rectangulation in Fig. 9 is generated through repeating a face adjacency spatial relation with aligned edges as shown in Fig. 10.

A label consisting of a small square indicates how the rule is applied among the symmetrical possibilities, although placement of the label and its symmetry mean that there remains a choice about applying the rule. However, it should be clear
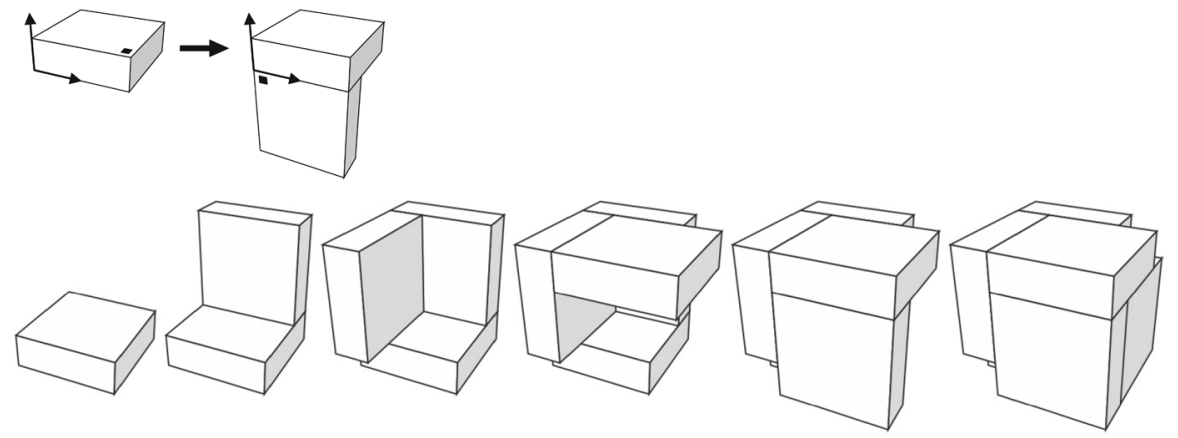

Fig. 10 Generating the pinwheel schema in Fig. 9 with a repeated shape rule (applied alternately under reflection)
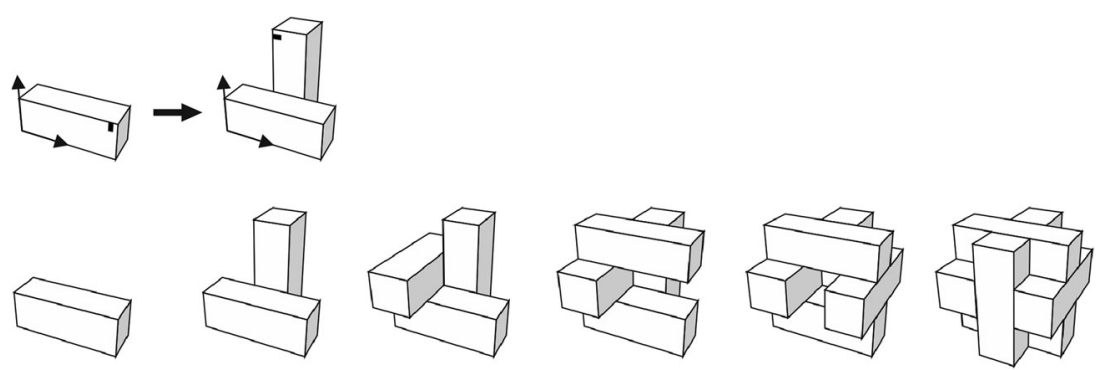

Fig. 11 Generating the pinwheel schema in Fig. 6 with a repeated shape rule 


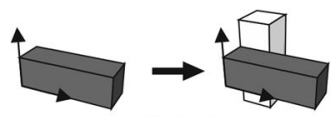

Rule 1

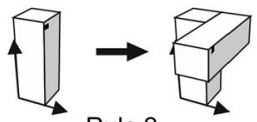

Rule 2

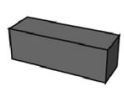

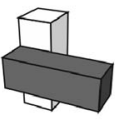

Rule 1

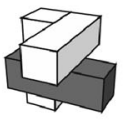

Rule 2

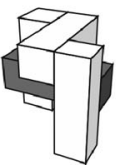

Rule 2

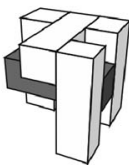

Rule 1

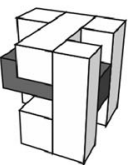

Rule 2

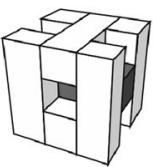

Rule 2

Fig. 12 Generating the pinwheel schema in Fig. 8 using two rules to wrap six cuboids around the central 'through' cuboid

which choice is used. The spatial relation is applied as a rule in a sequence of mirror reflections transformations. Symmetries of the shape include the rotations about the diagonal axes of the cube and a central inversion.

The pinwheel schema in Fig. 6, with six cuboids and eight 'internal' locked pinwheel joints is also generated by repeated application of a single rule (Fig. 11). The rule repetition leads to the identical $\mathrm{H}$ shaped internal maximal planes and the identical + shaped external planes. The rule essentially constructs one 3D pinwheel with three cuboids and then another which interlocks the first.

The third example of a pinwheel schema from Fig. 8 with seven cuboids and six internal 3D locked joints has three kinds of maximal plane which are $\square, \mathrm{H}$ or + shaped. Two rules generate this more complex schema (Fig. 12). As with the previous schema a locked joint is generated first around the central 'through' cuboid and further locked joints then created by wrapping further cuboids around the central one.

The three examples of pinwheel schema generated in this section form the basis of extended 3-D patterns analogous to the way that Serlio and Wallis constructed their extensions of the 2-D pinwheel. The 3-D pinwheel schemas present motifs for spatially extended patterns where a small number of simple spatial relations are repeated across the pattern. The next section analyses general classes of 3 -rectangulation in terms of dependencies in the numbers and types of joints, demonstrating the critical role that the 3-D locked pinwheel joints play general 3-rectangulations. These dependencies are expressed as counting formulae on spatial elements analogous to the Euler formula for counting the vertices edges, faces and holes in a polyhedron. 


\section{Counting Maximal Planes, Joints and Cuboids in 3-Rectangulations}

Compositions of face adjacent cuboids present diverse spatial arrangements. It is shown below how the numbers of joints of different types, maximal planes and cuboids, satisfy several formulae analogous to Euler's formula for polyhedra and more generally for polytopes.

Since each maximal plane has a boundary composed of lines along two orthogonal directions, the difference between the number of convex corners and concave corners on each maximal plane is $4(1-h)$ where $h$ is the number of holes in the maximal plane. A concave corner corresponds to a joint of type $C_{3}, C_{3}^{0}, C_{2}^{0}, C_{0}^{0}$ or $C_{1}^{0}$. A convex corner corresponds to a joint of type $X_{3}^{0}, X_{2}^{0}, X_{1}^{0}, X_{1}$ or $C_{1}^{0}$. One joint type, namely $C_{1}^{0}$, has one concave corner and two convex corners. The joints $C_{0}^{0}$ have no incident corners of cuboids and neither do the subset of the locked joints $C_{3}^{0}(a) \subseteq C_{3}^{0}$ which are 'external' on both sides. Other $C_{3}^{0}$ joints may have one incident cuboid corner or two if they are completely internal joints. The numbers of each type of joint are denoted by their corresponding type symbols.

If there are $P$ maximal planes with a total of $H$ holes then the total deficit between convex and concave corners on all maximal planes is

$$
4 P-4 H=\left\{X_{1}+3 X_{3}^{0}+2 X_{2}^{0}+X_{1}^{0}+C_{1}^{0}\right\}-\left\{3 C_{3}+3 C_{3}^{0}+2 C_{2}^{0}+C_{1}^{0}+C_{0}^{0}\right\}
$$

Counting the corners of 3-rectangles

$$
8 R=2\left\{X_{1}+C_{3}\right\}+\left\{C_{3}^{0}-C_{3}^{0}(a)+C_{2}^{0}+C_{1}^{0}\right\}+\left\{X_{3}^{0}+X_{2}^{0}+X_{1}^{0}\right\}
$$

For the polyhedron formed at the boundary with $F^{0}$ faces in which there are a total of $H^{0}$ holes the deficit between convex and concave corners on the faces is

$$
\begin{aligned}
4 F^{0}-4 H^{0}= & \left\{3 X_{3}^{0}+2 X_{2}^{0}+3 X_{1}^{0}+3 C_{3}^{0}+3 C_{3}^{0}(a)+2 C_{2}^{0}+2 C_{1}^{0}+2 C_{0}^{0}\right\} \\
& -\left\{X_{2}^{0}+C_{2}^{0}+C_{1}^{0}\right\}
\end{aligned}
$$

The boundary polyhedron with $F^{0}$ faces, $E^{0}$ edges and $V^{0}$ vertices has three edges at each joint except for joints $C_{0}^{0}$ with four incident edges and the joints $C_{3}^{0}(a) \subseteq C_{3}^{0}$ with six.

$$
\begin{aligned}
2 E^{0} & =3\left\{C_{3}^{0}+C_{3}^{0}(a)+C_{2}^{0}+C_{1}^{0}\right\}+3\left\{X_{3}^{0}+X_{2}^{0}+X_{1}^{0}\right\}+4 C_{0}^{0} V^{0} \\
& =C_{3}^{0}+C_{2}^{0}+C_{1}^{0}+C_{0}^{0}+X_{3}^{0}+X_{2}^{0}+X_{1}^{0}
\end{aligned}
$$

If the boundary polyhedron has $c^{0}$ connected parts with $g^{0}$ holes Euler's formula $\left(F^{0}-H^{0}\right)-E^{0}+V^{0}+C_{3}^{0}(a)=2 c^{0}-2 g^{0}$ gives

$$
X_{3}^{0}-X_{2}^{0}+X_{1}^{0}+C_{3}^{0}+C_{3}^{0}(a)-C_{2}^{0}-C_{1}^{0}-2 C_{0}^{0}=8 c^{0}-8 g^{0}
$$

Equations (1)-(3) yield

$$
C_{3}+C_{3}^{0}+1 / 2\left\{C_{2}^{0}-X_{3}^{0}-X_{2}^{0}\right\}=R-(P-H)+c^{0}-g^{0}
$$


For a 3-rectangulation with a single rectangular boundary

$$
C_{3}=(R-1)-(P-H)+6
$$

For a 3-rectangulation with all maximal planes as rectangles

$$
X_{3}^{0}+X_{2}^{0}=2(P-R)-2 c^{0}
$$

This counting assumes face adjacencies between 3 -rectangles. If line- and pointadjacencies are present then they can be derived by adjusting face-adjacencies to create alignments. Adding line-adjacency joints of type $C_{3}^{0}$ and type $C_{2}^{0}$ joint to the allowable joints maintains the validity of Eq. (4) since joints of types $C_{1}^{0}, C_{0}^{0}$ and $X_{1}^{0}$ are not included in (4). A simple example would be a layered 'octahedron' where each layer is edge adjacent to its neighbours above and below, and with a void interior. There is a single cube at the top and bottom and three layers between. This configuration counts with

$$
C_{2}^{0}=16, X_{3}^{0}=24, R=14, P=22, H=2, c^{0}-g^{0}=2 .
$$

The counting of joint types, cuboids and holes developed above is now applied to the three examples of pinwheel schema in Figs. 10, 11 and 12. This shows how joints are counted in each case as well as checking the general result in Eq. (3).

For the schema in Fig. 10: $C_{3}^{0}=2, C_{1}^{0}=6, X_{3}^{0}=12, X_{1}=12, X_{1}^{0}=2, R=$ $6, P=12, c^{0}=2$. Equation (4) counts these as RHS $=-4=$ LHS.

For the schema in Fig. 11: $C_{3}^{0}=8, C_{1}^{0}=24, X_{3}^{0}=24, X_{1}=12, R=6, P=$ $12, c^{0}=2$. Equation (4) counts these as RHS $=-4=$ LHS.

For the schema in Fig. 12: $C_{3}^{0}=8, C_{1}^{0}=24, X_{3}^{0}=16, X_{1}=8, R=7, P=$ $12, H=4, c^{0}=1$. Equation (4) counts these as RHS $=0$, LHS $=0$.

Line and point adjacencies allow maximal planes to intersect along lines not on their boundaries. However, joints are restricted in the above analysis so that no more than two cuboids are adjacent on a single line segment or at a point.

\section{Conclusion}

Unlocked 3-rectangulations are essentially two-dimensional when described by their generative rules (Krishnamurti and Earl 1998). These are subdivision rules applied to the 2-rectangulation cross sections and show how the unlocked 3-rectangulations are local variants of layered 2-dimensional layouts.

Locked 3-rectangulations have more complex generative rules. The pinwheel schemas from the elemental locked joint, to its composite configurations described above, encapsulate this three-dimensional complexity. However, there are other ways to generate general 3-rectangulations that only rely on these schemas indirectly, and present less constrained possibilities.

One way of developing such rules depends on 'puncturing' planes with holes. As seen above it is these holes that create the concave corners in the maximal planes indicative of pinwheel patterns. Each maximal plane is effectively divided into rectangles by the incident orthogonal planes. Internal maximal planes are divided in two ways, one on each side of the plane. The plane segments in the boundary of the 
3-rectangulation are divided only from the inside. A rule aligns two equal configurations of rectangles, one on each side an internal plane and then the extra rule 'knocks though' the plane dividing them. The rule identifies the aligned rectangles, removing the corresponding parts of the dividing plane and merging planes and rectangles. As these additional rules are applied, maximal planes will take on more complex boundaries, possibly with holes.

These kinds of rules construct the 3-rectangulation and maintain at each stage of generation the essential properties of joint type and rectangular boundary. With more general configurations of 3-rectangles, other generative specifications are possible. Indeed there seems no barrier to straightforward composition by adding one rectangle at a time, face adjacent to one or more existing rectangles. Rule application is constrained so that no more than two rectangles are incident on a single line segment. Further constraints ensure all rectangles are disjoint. A rule may create any number of face adjacencies. For example, a vacant cuboid hole can be filled by a new 3-rectangulation.

These kinds of rules for constructing general 3-rectangulations are trivial but their constraints complex, especially the requirement not to overlap rectangles in the face adjacent 3-rectangulations. However, as with many constraints and rules, compositions such as the Schroeder House, and perhaps more boldly in the intersecting volumes of constructivist artists such as Chernikov (Cooke 1989) these constraints and rules are broken especially in apparently intersecting rectangular slabs-whether architectural volumes or walls. This fluidity of the intersections of rectangular slabs as constructional elements and the flow of space through intersections of architectural volumes echo each other.

The wide range of spatial relations exploited in these works can be accommodated through extensions of the notations and counting patterns presented in this paper. These will be developed in a subsequent paper where overlapping and intersections of cuboids and volumes give new types of joint on the maximal planes.

One more degree of complementarity is perhaps worth a mention. The two regimes for generation; addition of face adjacent cuboids with constraints and the subdivision of rectangles are two complementary ways to 'see' configurations as well as to generate them. The flows between composition and division, between planes (or structural building elements) and volumes (or architectural spaces) are exemplified in the face adjacent rectangular configurations considered here.

The pinwheel schemas presented here demonstrate the essential three-dimensional nature of spatial configurations on an orthogonal grid. Their analysis helps in understanding a long history of explorations in spatial composition in design and architecture. Their generative specification offers a tool for further exploration, construction and explanation.

Acknowledgments All images are by the authors, unless otherwise stated

\section{References}

Brittain-Catlin, T. 2004. Pugin's English Residential Architecture in its Context. Ph.D. thesis, Cambridge University. 
Cooke, C. A. C. 1989. ed. Fantasy and Construction-Iakov Chernikhov. AD magazine, vol 59 no 7-8, London.

Dettingmeijer, R., van Thoor, M-T. and van Zijl, I. (eds.) 2010. Rietveld's Universe. Rotterdam: NAI.

Earl, C. F. 1978. Joints in two- and three-dimensional rectangular dissections. Environment and Planning B 5(2): 179-187.

Krishnamurti, R. and Earl, C. 1998. Densely packed rectangulations. Environment and Planning B, Planning and Design 25: 773-787.

MacCormac, R. C. 1974. Froebel's kindergarten gifts and the early work of Frank Lloyd Wright. Environment and Planning B 1(1): 29-50.

March, L. and Steadman, P. 1971. Geometry of Environment. London: RIBA.

Serlio, S. 1545. Il primo libro d'architettura, di Sebastiano Serlio. Paris.

Stiny, G. 1980. Kindergarten grammars: designing with Froebel's building gifts Environment and Planning B 7(4): 409-462.

Van Doesburg, T. 1969. Principles of Neo-Plastic Art. London: Lund Humphries.

Wallis, J. 1670. Mechanica. London.

Yeomans, D. 1997. The Serlio floor and its derivations. Architectural Research Quarterly, 2:74-83.

Christopher F. Earl (BA, M.Sc. Mathematics, Ph.D. Design) is Professor of Engineering Design at the Open University, UK, since 2000. His primary area of research is in generative synthesis in Design, particularly relating to shape and function. His other research includes modelling and planning design processes. From 1991-2000 he was in the Engineering Design Centre, at Newcastle University, where his research concentrated on integration of design and manufacture, particularly in planning complex, engineer-to-order products.

Iestyn Jowers (B.Sc., M.Sc., Ph.D. Design) is a Research Fellow in the Design Group at the Open University, UK. His primary area of research is in the role of shapes in design processes, including investigations into shape computations and shape grammars, generative design, additive manufacturing and computational making. Prior to joining the Open University in 2013 he worked as a post-doctoral research fellow at TU Munich, University of Leeds, and University of Cambridge. 